\title{
Importance of timely monitoring of seasonal influenza vaccine effectiveness
}

RG Pebody ${ }^{1}$, K Mølbak ${ }^{2}$

1. Public Health England, London, United Kingdom

2. Statens Serum Institut, Copenhagen, Denmark

Correspondence: Richard G Pebody (richard.pebody@phe.gov.uk)

Citation style for this article:

Pebody RG, Mølbak K. Importance of timely monitoring of seasonal influenza vaccine effectiveness. Euro Surveill. 2016;21(16):pii=30209. DOI: http://dx.doi. org/10.2807/1560-7917.ES.2016.21.16.30209

Article submitted on 13 April 2016 / accepted on 20 April 2016 / published on 21 April 2016

Seasonal influenza vaccination programmes represent one the largest components of national immunisation programmes in many industrialised countries with a wide range of target groups in the population. These programmes target groups at higher risk of severe disease including the elderly, those with underlying clinical risk factors and pregnant women in many European countries [1]. Additionally many countries offer vaccines to healthcare workers and some to healthy children [1]. The rationale for vaccinating the latter is to both directly protect the vaccinated persons themselves by reducing the spread of infection and indirectly protect other groups at higher risk of severe disease whether that is in the local community or the hospital where they work.

Due to changes in the dominant circulating strains each season and the limited length of protection [2] afforded by the current generation of influenza vaccines, countries undertake annual vaccination campaigns. These time-limited programmes are usually conducted in the period just prior to the start of the influenza season to maximise population protection. Annual public health monitoring of the effectiveness of seasonal influenza vaccine has now become well established in North America, Europe and Australasia to complement existing virological surveillance and characterisation of circulating strains. Countries use the test-negative case-control approach through established sentinel primary care swabbing networks or comparable data sources, with many countries undertaking mid-season vaccine effectiveness (VE) estimates [3]. These earlyseason estimates are important for several reasons. Firstly, together with available virus characterisation data, they provide an early indication of how well the current season's vaccine is (or is not) matched to the circulating strains: this enables public health measures to be refined if necessary e.g. the use of antivirals to further reduce the health impact of influenza. VE measures combined with estimates and projections of number of hospital admissions related to influenza are also important for healthcare service planning and situational awareness. Finally, the information from these mid-season VE estimates is provided to the World Health Organization (WHO) twice-yearly convened influenza vaccine composition meeting by the Global Influenza Vaccine Effectiveness collaboration together with virological characterisation and serological data [4]. This group recommends the content of the seasonal influenza vaccine for the northern and southern hemispheres that vaccine manufacturers need to produce ready for the vaccine campaigns six months later. These estimates are importantly provided independent of the vaccine manufacturers, who are required to submit safety and effectiveness data as part of recently introduced European Medicines Agency requirements [5].

Two papers in this week's edition of Eurosurveillance highlight further the importance of this timely seasonal influenza VE monitoring in optimising seasonal influenza vaccination strategies $[6,7$,$] while a third$ addresses pandemic vaccination strategies in the Nordic countries, 2009 [8]. The more ready availability of epidemiological VE data has provided the WHO committee with further and timelier insights into the match between circulating and vaccine strains and enhances its ability to make the best recommendations possible about the vaccine strain composition for the forthcoming season using epidemiological, virological and serological data. The first paper by Leung et al., a systematic review over almost a decade, reinforces this point, with the article demonstrating the usual reliability of these early-season VE estimates when compared to the final end-of-season estimates. The authors also demonstrate that in the majority of studies, the midseason VE estimates were within $10 \%$ of the final endof-season estimate, with the vast bulk of the interim estimates provided ahead of the WHO influenza vaccine composition meeting. The paper also highlights 
the importance of ensuring a standard approach to enhance the comparability between mid-and end-ofseason VE, and that protocols need to meet this aim.

The second paper by Kissling et al. from the European I-MOVE network examines the important question of whether there is any evidence of intra-seasonal waning of VE over the period from 2010/11 to 2014/15. They demonstrate evidence of consistent reductions in VE against $\mathrm{A}\left(\mathrm{H}_{3} \mathrm{~N}_{2}\right)$ to $0 \%$ by $>$ three months after vaccination across all seasons examined; with smaller reductions for influenza $B$ and a stable VE against $A\left(\mathrm{H}_{1} \mathrm{~N}_{1}\right)$ pdmog throughout the season. They discuss potential explanations for these observations in particular disentangling intra-seasonal waning of vaccine-derived immunity versus changes in circulating strains which may be antigenically mismatched later in the season. Interestingly the waning findings are mainly restricted to $\mathrm{A}\left(\mathrm{H}_{3} \mathrm{~N}_{2}\right)$. This subtype is recognised to be challenging as a vaccine target, and which mainly results in health impact in the elderly. From the paper by Leung et al. [6], the overall population impact of this 'waning' of VE can be seen when comparing the mid and end-ofseason estimates, reinforcing the findings from Kissling et al. [7]. The reductions in VE on the population level are likely to be more apparent when $\mathrm{A}\left(\mathrm{H}_{3} \mathrm{~N}_{2}\right)$ circulates later in the season, as was the case in $2013 / 14$, when a number of countries reported evidence of reductions in $A\left(\mathrm{H}_{3} \mathrm{~N}_{2}\right)$ VE later in the season.

Whatever the explanation for these observations, the findings of intra-seasonal waning raise important questions about what the optimal intervention strategy is. The authors propose undertaking campaigns later in the season. Practically, this would be a challenging policy to implement, particularly in larger temperate countries. With the timing of influenza activity so variable each year and the season usually lasting at least 6 to 8 weeks; campaigns in the northern hemisphere need to be largely completed by end of December before the season starts. As vaccine is only available usually from October onwards and the delivery of the annual campaign requires several weeks of intensive vaccination activity (including two weeks for protection to be acquired), there is little flexibility in timing, without taking real risks of not providing the population protection required before influenza circulation starts. What strategies might be employed otherwise? Even in an optimal scenario with a good match between the circulating influenza strain and the vaccine, and with a timing of the season in favour of the vaccine, the effectiveness is less than other vaccines offered in the childhood vaccination programmes. Although there is a clear need for new and better influenza vaccines, possibly targeting conserved antigens; there is also a need to identify which of the existing available influenza vaccines e.g. adjuvanted and high dose inactivated or quadrivalent versus trivalent, might provide optimal protection in key target groups, particularly the elderly where the impact of $\mathrm{A}\left(\mathrm{H}_{3} \mathrm{~N}_{2}\right)$ is usually greatest. How these vaccines might be used better should also be considered as highlighted by Kissling et al., VE depends on age, and although the sample size of their study was not big enough to determine if there was waning immunity in smaller age strata, one question might be if waning vaccine-derived immunity against influenza $A\left(\mathrm{H}_{3} \mathrm{~N}_{2}\right)$ is less of a problem in the younger age groups. This would be supportive of another intervention strategy, where the primary focus would be preventing the spread of influenza to groups at higher risk of severe disease by vaccinating children. This approach of trying to provide both direct and indirect population protection is currently being introduced in the United Kingdom through a new vaccination programme of healthy children with live attenuated influenza vaccine. As also mentioned by Kissling et al., the current season influenza VE may vary by prior influenza vaccine history, and there is a need to understand this better to ensure optimal intervention strategies are developed. This strategy is also supported in a third paper by Gil Cuesta et al. [8] also published in this issue, that demonstrates lower cumulative rates of influenza $\mathrm{A}\left(\mathrm{H}_{1} \mathrm{~N}_{1}\right)$ pdmog infection in the influenza season following the 2009 pandemic in the four of five Nordic countries with higher pandemic vaccine coverage in the wider general population, including children. This indicates that in the assessment of impact of vaccination strategy, it may be important to look at more than one season, possibly taking type of vaccine and age-group targeted into account.

It is also important to note that there are other interventions than vaccines. Public health authorities need to consider how the use of antiviral drugs might be optimised to further reduce morbidity and mortality particularly when influenza seasons are unusually late. Finally, behavioural measures such as hand hygiene, avoiding close contact to sick persons, staying home when sick and cough etiquette are measures that can contribute to prevention of the spread of influenza throughout the influenza season $[9,10]$.

\section{Conflict of interest}

Richard Pebody and Kåre Mølbak are both members of the I-MOVE+ network. KM is a co-author on one of the published papers highlighted.

Authors' contributions

Both authors contributed to writing this editorial.

References

1. Mereckiene J, Cotter S, O'Flanagan D, VENICE III Consortium. National seasonal influenza vaccination survey for 2012-13 influenza season in EU/EEA (provisional data). Available from: http://venice.cineca.org/VENICE_Seasonal_ Influenza_2012-13_v10.pdf

2. Kissling $E$, Valenciano $M$, Larrauri A, Oroszi B, Cohen JM, Nunes $B$, et al. Low and decreasing vaccine effectiveness against influenza $A\left(\mathrm{H}_{3}\right)$ in $2011 / 12$ among vaccination target groups in Europe: results from the I-MOVE multicentre casecontrol study. Euro Surveill. 2013;18(5):20390.

3. Skowronski DM, Masaro C, Kwindt TL, Mak A, Petric M, Li Y, et al. Estimating vaccine effectiveness against laboratoryconfirmed influenza using a sentinel physician network: 
results from the $2005-2006$ season of dual $A$ and $B$ vaccine mismatch in Canada. Vaccine. 2007;25(15):2842-51. Epub 2006 Oct 16.

4. World Health Organization (WHO) Recommended composition of influenza virus vaccines for use in the 2016-2017 northern hemisphere influenza season. Geneva: WHO; Feb 2016. Available from: http://www.who.int/influenza/vaccines/virus/ recommendations/201602 recommendation.pdf?ua $=1$

5. European Medicines Agency (EMA). Risk-management plans. London: EMA. Available from: http://www.ema.europa.eu/ ema/index.jsp?curl=pages/regulation/document_listing/ document_listing_000360.jsp

6. Leung VK, Cowling BJ, Feng S, Sullivan SG. Concordance of interim and final estimates of influenza vaccine effectiveness: a systematic review. Euro Surveill. 2016;21(16):pii=30202. DOI: 10.2807/1560-7917.ES.2016.21.16.30202

7. Kissling E, Nunes B, Robertson C, Valenciano M, Reuss A, Larrauri A, et al. I-MOVE multicentre case-control study $2010 / 11$ to $2014 / 15$ : Is there within-season waning of influenza type/subtype vaccine effectiveness with increasing time since vaccination? . Euro Surveill. 2016;21(16):pii=30201. DOI: 10.2807/1560-7917.ES.2016.21.16.30201

8. Cuesta JG, Aavitsland P, Englund H, Gudlaugsson Ó, Hauge $\mathrm{SH}$, Lyytikäinen $\mathrm{O}$, et al. Pandemic vaccination strategies and influenza severe outcomes during the influenza $A\left(\mathrm{H}_{1} \mathrm{~N}_{1}\right)$ pdmog pandemic and the post-pandemic influenza season: the Nordic experience. Euro Surveill. 2016;21(16):pii=30208. DOI: 10.2807/1560-7917.ES.2016.21.16.30208

9. Rainwater-Lovett K, Chun K, Lessler J. Influenza outbreak control practices and the effectiveness of interventions in long-term care facilities: a systematic review Influenza Other Respir Viruses. 2014 Jan;8(1):74-82. doi: DOI: 10.1111/irv.12203 . Epub 2013 Nov 7. Review. PMID: 24373292

10. Torner N, Soldevila N, Garcia JJ, Launes C, Godoy P, Castilla $J$, et al. Effectiveness of non-pharmaceutical measures in preventing pediatric influenza: a case-control study. BMC Public Health. 2015;15:543. DOI: 10.1186/s12889-015-1890-3

\section{License and copyright}

This is an open-access article distributed under the terms of the Creative Commons Attribution (CC BY 4.0) Licence. You may share and adapt the material, but must give appropriate credit to the source, provide a link to the licence, and indicate if changes were made.

This article is copyright of the authors, 2016. 